\title{
Convergence of subdivision schemes on Riemannian manifolds with nonpositive sectional curvature
}

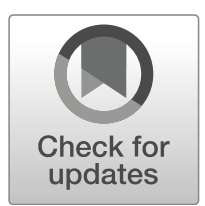

\section{Svenja Hüning ${ }^{1}$ Johannes Wallner ${ }^{1}$}

Received: 29 June 2018 / Accepted: 27 March 2019 /

Published online: 2 May 2019

(C) The Author(s) 2019

\section{Abstract}

This paper studies well-definedness and convergence of subdivision schemes which operate on Riemannian manifolds with nonpositive sectional curvature. These schemes are constructed from linear ones by replacing affine averages by the Riemannian centre of mass. In contrast to previous work, we consider schemes without any sign restriction on the mask, and our results apply to all input data. We also analyse the Hölder continuity of the resulting limit curves. Our main result states that if the norm of the derived scheme (resp. iterated derived scheme) is smaller than the corresponding dilation factor then the adapted scheme converges. In this way, we establish that convergence of a linear subdivision scheme is almost equivalent to convergence of its nonlinear manifold counterpart.

Keywords Refinement processes - Riemannian geometry - Geodesic averaging · Hölder continuity

Mathematics Subject Classification (2010) 41A25 - 65D05 · 65D17

\section{Introduction}

Linear stationary subdivision schemes are well-studied regarding their properties of convergence and smoothness, see for example [2]. Over the last years, linear refinement rules were transferred to nonlinear geometries, and subdivision algorithms have been applied to data coming from surfaces, Lie groups or Riemannian

\footnotetext{
Communicated by: Tom Lyche

Svenja Hüning

huening@tugraz.at

Johannes Wallner

j.wallner@tugraz.at

1 Institut f. Geometrie, TU Graz, Kopernikusgasse 24, 8010 Graz, Austria
} 
manifolds. Different methods have been introduced to extend linear refinement algorithms to manifold-valued data. Examples are the log-exp-analogue of a linear scheme $[4,18]$, geodesic averaging processes or the so-called projection analogue, see [11] for an overview.

Many results on convergence of nonlinear refinement processes are based on the so-called proximity conditions introduced in [21]. These convergence results unfortunately only apply to 'dense enough' input data.

If convergence is assumed, many nonlinear constructions yield $C^{1}$ and $C^{2}$ smoothness, see, e.g. [12, 19, 22]. The full smoothness of linear schemes is reproduced only if certain ways of constructing nonlinear schemes from linear ones are employed [11, 24].

Returning to the question of convergence of nonlinear subdivision schemes, some results apply to all input data. One can show convergence, e.g. for interpolatory schemes in Riemannian manifolds [20] or schemes defined by binary geodesic averaging $[7,8]$. If one restricts to special geometries, more general classes of schemes can be shown to converge for all input data, e.g. schemes with nonnegative mask in Cartan-Hadamard metric spaces have been treated by [9, 10]. In this general setting, which goes beyond smooth manifolds, the coefficients of the scheme's mask are interpreted as probabilities.

In this paper, we prove convergence of subdivision schemes in complete Riemannian manifolds with sectional curvature $K \leqslant 0$. We generalise earlier work, in particular Theorem 5 of [23] which can only be applied to schemes with nonnegative mask. To extend linear refinement rules to manifold-valued data, we use the Riemannian centre of mass [15]. Such refinement rules have been investigated by [11] regarding their smoothness, and in [23] with regard to convergence. A synonym for 'Riemannian centre of mass' which has been used is weighted geodesic average.

The paper is organised as follows. First, we recall some facts about linear subdivision schemes and their nonlinear counterparts. In particular, we introduce a Riemannian analogue $T$ of a linear scheme $S$ and show that it is well-defined in Cartan-Hadamard manifolds. In Section 4, we prove that $T$ is contractive and displacement-safe, in the terminology introduced in [8]. Afterwards, we deduce our main result which states that if

$$
\frac{1}{N^{m}}\left\|S^{m *}\right\|<1, \quad \text { for some } m=1,2, \ldots,
$$

then a Riemannian analogue of $S^{m}$ converges to a continuous limit curve. Here, $N$ denotes the dilation factor and $S^{*}$ is the derived scheme. Next, we analyse the Hölder regularity of the limit curves. Moreover, we describe how to extend our results to a wider class of manifolds by dropping the simple connectivity required for Cartan-Hadamard manifolds. The last section presents some examples. For the reader's convenience, some selected proofs have been moved to the Appendix. 


\section{Subdivision schemes}

\subsection{Linear subdivision schemes}

A linear subdivision scheme $S$ maps a sequence of points $\left(x_{i}\right)_{i \in \mathbb{Z}}$ lying in a linear space to a new sequence of points $\left(S x_{i}\right)_{i \in \mathbb{Z}}$ using the rule

$$
S x_{i}=\sum_{j \in \mathbb{Z}} a_{i-N j} x_{j} .
$$

Here, $N \in \mathbb{N}$ is the dilation factor. We require $N \geqslant 2$, but the usual case is $N=2$. Throughout the paper, we assume that the sequence $a_{\ell}, \ell \in \mathbb{Z}$, called the mask of the refinement rule, has compact support. This means that $a_{\ell} \neq 0$ only for finitely many $\ell$. It turns out that the condition

$$
\sum_{j \in \mathbb{Z}} a_{i-N j}=1 \text { for all } i
$$

(affine invariance) is necessary for the convergence of linear subdivision schemes, see [8] and [2] for an overview. From now on, we make the assumption that all subdivision schemes are affine invariant.

To simplify notation, we initially consider only binary refinement rules, i.e. rules with dilation factor $N=2$. Then, we can write the refinement rule in the following way:

$$
(S x)_{2 i}=\sum_{j=-m}^{m+1} \alpha_{j} x_{i+j} \quad \text { and } \quad(S x)_{2 i+1}=\sum_{j=-m}^{m+1} \beta_{j} x_{i+j},
$$

with $m \in \mathbb{N}$ and coefficients $\alpha_{j}, \beta_{j}$ such that

$$
\sum_{j=-m}^{m+1} \alpha_{j}=\sum_{j=-m}^{m+1} \beta_{j}=1
$$

For example, Chaikin's algorithm [3], which is given by the mask $\left(a_{-2}, \ldots, a_{1}\right)=$ $\left(\frac{1}{4}, \frac{3}{4}, \frac{3}{4}, \frac{1}{4}\right)$, can be written as

$$
(S x)_{2 i}=\frac{3}{4} x_{i}+\frac{1}{4} x_{i+1} \quad \text { and } \quad(S x)_{2 i+1}=\frac{1}{4} x_{i}+\frac{3}{4} x_{i+1} .
$$

Subdivision schemes satisfying $(S x)_{2 i}=x_{i}$ are called interpolatory. For example, the well-known four-point scheme is defined by

$$
(S x)_{2 i}=x_{i} \quad \text { and } \quad(S x)_{2 i+1}=-\omega x_{i-1}+\left(\frac{1}{2}+\omega\right) x_{i}+\left(\frac{1}{2}+\omega\right) x_{i+1}-\omega x_{i+2},
$$

for some parameter $\omega$, see [6]. The next example will be our main example throughout the text. 
Example 1 We consider a non-interpolatory subdivision scheme with negative mask coefficients. Taking averages of the four-point scheme with parameter $\omega=\frac{1}{16}$ and Chaikin's scheme yields

$$
\begin{aligned}
(S x)_{2 i} & =-\frac{1}{32} x_{i-1}+\frac{21}{32} x_{i}+\frac{13}{32} x_{i+1}-\frac{1}{32} x_{i+2}, \\
(S x)_{2 i+1} & =-\frac{1}{32} x_{i-1}+\frac{13}{32} x_{i}+\frac{21}{32} x_{i+1}-\frac{1}{32} x_{i+2}
\end{aligned}
$$

\subsection{The Riemannian analogue of a linear subdivision scheme}

We recall the extension of a linear subdivision scheme to manifold-valued data with the help of the Riemannian centre of mass as shown in [11]. This generalisation of the concept of affine average is quite natural in the sense that we only replace the Euclidean distance by the Riemannian distance. The construction requires to introduce some notation. We denote the Riemannian inner product by $\langle\cdot, \cdot\rangle=|\cdot|^{2}$ on a Riemannian manifold $M$. The Riemannian distance $\operatorname{dist}(x, y)$ between two points $x, y \in M$ is given by

$$
\operatorname{dist}(x, y):=\inf _{\gamma} \int_{a}^{b}|\dot{\gamma}(t)| d t,
$$

where $\gamma:[a, b] \rightarrow M$ is a curve connecting points $\gamma(a)=x$ and $\gamma(b)=y$. Consider the weighted affine average

$$
x^{*}=\sum_{j=0}^{n} \alpha_{j} x_{j}
$$

of points $x_{j} \in \mathbb{R}^{d}$ w.r.t. weights $\alpha_{j} \in \mathbb{R}$, satisfying $\sum \alpha_{j}=1$. It can be characterised as the unique minimum of the function

$$
g_{\alpha}(x)=\sum_{j=0}^{n} \alpha_{j}\left|x-x_{j}\right|^{2} .
$$

We transfer this definition to Riemannian manifolds by replacing the Euclidean distance by the Riemannian distance. Let

$$
f_{\alpha}(x)=\sum_{j=0}^{n} \alpha_{j} \operatorname{dist}\left(x, x_{j}\right)^{2} .
$$

We call the minimizer of this function the Riemannian centre of mass and denote it by

$$
x^{*}=\operatorname{av}(\alpha, x) .
$$

Note that in general the Riemannian centre of mass is only well-defined locally. It is the aim of the present paper to identify settings where the average is globally welldefined. We extend the linear subdivision rule (2) to manifold-valued data by defining

$$
(T x)_{2 i}=\operatorname{av}(\alpha, x) \quad \text { and } \quad(T x)_{2 i+1}=\operatorname{av}(\beta, x) .
$$

Definition 2 We call $T$ the Riemannian analogue of the linear subdivision scheme $S$. 


\section{The Riemannian centre of mass in Cartan-Hadamard manifolds}

Cartan-Hadamard manifolds, and more generally manifolds with nonpositive sectional curvature, are a class of geometries where the Riemannian average can be made globally well-defined. Let $M$ be a Cartan-Hadamard manifold, i.e. a simply connected, complete Riemannian manifold with sectional curvature $K \leqslant 0$. To show well-definedness of geodesic averages, we have to clarify the global existence and uniqueness of a minimizer of the function

$$
f_{\alpha}(x)=\sum_{j=-m}^{m+1} \alpha_{j} \operatorname{dist}\left(x_{j}, x\right)^{2}, \quad \text { with } \quad \sum_{j} \alpha_{j}=1
$$

and $x_{j} \in M$. A local answer to this question is not difficult, see for example [17]. The global well-definedness in case $\alpha_{j} \geqslant 0$ is shown in [16]. Hanne Hardering gave another proof of the global existence in [13]. We are mainly interested in the result she gave in Lemma 2.3. of [13] which we formulate as

Lemma 3 (H. Hardering, [13]) The function $f_{\alpha}$ has at least one minimum. Moreover, there exists $r>0$ (depending on the coefficients $\alpha_{j}$ and the distances of the points $x_{j}$ from each other) such that all minima of $f_{\alpha}$ lie inside the compact ball $\overline{B_{r}\left(x_{0}\right)}$.

To prove that the function $f_{\alpha}$ has a unique minimum, we generalise a statement of Hermann Karcher [15]. It turns out that we can use arguments similar to his by splitting $\sum_{j=-m}^{m+1} \alpha_{j} \operatorname{dist}\left(x_{j}, x\right)^{2}$ into two sums depending on whether the corresponding coefficient is negative or not. Before we introduce the general notation used throughout the text, we illustrate the idea by means of Example 1.

Example 4 Consider the subdivision rule defined by the coefficients $\alpha_{j}$ and $\beta_{j}$ of Example 1. Define $f_{\alpha}$ according to (7) by

$$
f_{\alpha}(x)=\sum_{j=-1}^{2} \alpha_{j} \operatorname{dist}\left(x_{j}, x\right)^{2},
$$

with $\left(\alpha_{-1}, \ldots, \alpha_{2}\right)=\left(-\frac{1}{32}, \frac{21}{32}, \frac{13}{32},-\frac{1}{32}\right)$. We sort these coefficients in two groups depending on whether they are positive or not.

It is convenient to define $\alpha_{+}=\frac{21}{32}+\frac{13}{32}=\frac{34}{32}$ and $\alpha_{-}=\left|-\frac{1}{32}\right|+\left|-\frac{1}{32}\right|=\frac{2}{32}$. We split the interval $\left[0, \alpha_{+}+\alpha_{-}\right]$in four subintervals whose length coincides with the values $\left|\alpha_{j}\right|$ (but in a different order). We define the function $\sigma:\left[0, \alpha_{+}+\alpha_{-}\right] \rightarrow$ $\{-1,0,1,2\}$ by

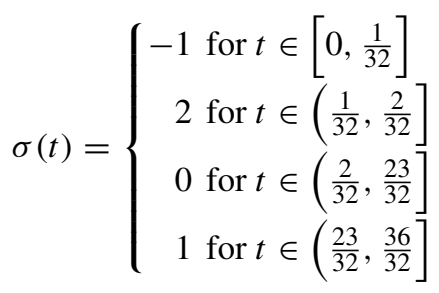


and see that

$$
f_{\alpha}(x)=\sum_{j=-1}^{2} \alpha_{j} \operatorname{dist}\left(x_{j}, x\right)^{2}=-\int_{0}^{\alpha_{-}} \operatorname{dist}\left(x_{\sigma(t)}, x\right)^{2} d t+\int_{\alpha_{-}}^{\alpha_{-}+\alpha_{+}} \operatorname{dist}\left(x_{\sigma(t)}, x\right)^{2} d t .
$$

In the general case, we need the following notation to eventually rewrite the function in (7) as the sum of two integrals (Fig. 1). We begin to sort our coefficients in two groups by defining two index sets

$$
I_{-}^{\alpha}:=\left\{j \mid \alpha_{j}<0\right\}, \quad I_{+}^{\alpha}:=\left\{j \mid \alpha_{j} \geqslant 0\right\} .
$$

We describe these sets as

$$
I_{-}^{\alpha}=\left\{j_{1}, \ldots, j_{n}\right\}, \quad I_{+}^{\alpha}=\left\{j_{n+1}, \ldots, j_{2 m+2}\right\},
$$

with $j_{1}<\ldots<j_{n}$ and $j_{n+1}<\ldots<j_{2 m+2}$ for $n \in\{1, \ldots, 2 m+2\}$ and $j_{i} \in$ $\{-m, \ldots, m+1\}$. If $I_{-}^{\alpha}=\emptyset$, we set $n=0$ and $I_{+}^{\alpha}=\{-m, \ldots, m+1\}$. Let

$$
\alpha_{+}=\sum_{j \in I_{+}^{\alpha}} \alpha_{j}, \quad \alpha_{-}=\sum_{j \in I_{-}^{\alpha}}\left|\alpha_{j}\right|, \quad \beta_{+}=\sum_{j \in I_{+}^{\beta}} \beta_{j}, \quad \beta_{-}=\sum_{j \in I_{-}^{\beta}}\left|\beta_{j}\right| .
$$

Assumption (3) implies that

$$
\alpha_{+}-\alpha_{-}=\beta_{+}-\beta_{-}=1
$$

We are now able to rewrite the function $f_{\alpha}$ in terms of two integrals

$$
f_{\alpha}(x)=\sum_{j=-m}^{m+1} \alpha_{j} \operatorname{dist}\left(x_{j}, x\right)^{2}=\left(-\int_{0}^{\alpha_{-}}+\int_{\alpha_{-}}^{\alpha_{-}+\alpha_{+}}\right) \operatorname{dist}\left(x_{\sigma(t)}, x\right)^{2} d t
$$

with the function $\sigma:\left[0, \alpha_{+}+\alpha_{-}\right] \rightarrow\{-m, \ldots, m+1\}$ given as follows. It is constant in each of the successive intervals of length $\left|\alpha_{j_{1}}\right|,\left|\alpha_{j_{2}}\right|, \ldots,\left|\alpha_{j_{2 m+2}}\right|$ which tile the interval $\left[0, \alpha_{+}+\alpha_{-}\right]$. Its value in the $k$-th interval is given by the integer $j_{k}$. The values at subinterval boundaries are not relevant. We note that the first part of the definition of $\sigma$ represents the summands of (7) corresponding to

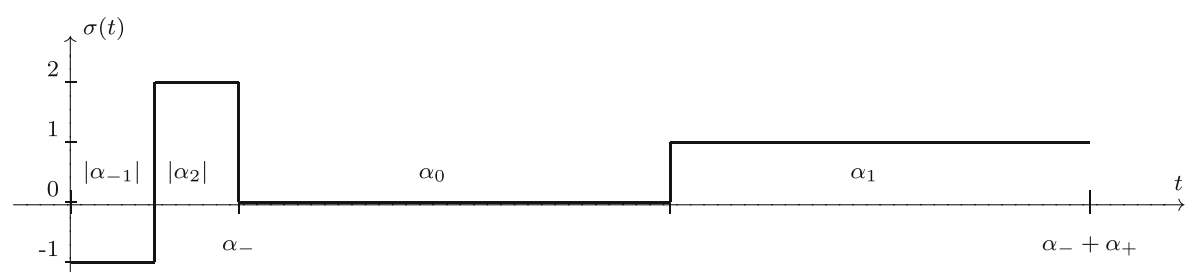

Fig. 1 Construction of the index selection function $\sigma$ on basis of the sequence $\left(\alpha_{j}\right)_{j=-1}^{2}$ with $\alpha_{-1}, \alpha_{2}<0$, $\alpha_{0}, \alpha_{1}>0$ 
coefficients of $I_{-}^{\alpha}$ whereas the second part represents the coefficients corresponding to $I_{+}^{\alpha}$.

Using the representation of the function $f_{\alpha}$ given in (9), we can state

Lemma 5 In a Cartan-Hadamard manifold, the gradient of the function $f_{\alpha}$ is given by the formula

$$
\frac{1}{2} \operatorname{grad} f_{\alpha}(x)=\int_{0}^{\alpha_{-}} \exp _{x}^{-1} x_{\sigma(t)} d t-\int_{\alpha_{-}}^{\alpha_{-}+\alpha_{+}} \exp _{x}^{-1} x_{\sigma(t)} d t,
$$

where exp denotes the Riemannian exponential map. Furthermore, we have

$$
\frac{d^{2}}{d s^{2}} f_{\alpha}(\gamma(s)) \geqslant 2\langle\dot{\gamma}(s), \dot{\gamma}(s)\rangle
$$

for any geodesic $\gamma:[0,1] \rightarrow M$.

The proof of this lemma is contained in the Appendix. It is mainly based on the proof of Theorem 1.2. in [15]. We sum up the results of the two lemmas above to state the main result of this section.

Theorem 6 In a Cartan-Hadamard manifold $M$, the function

$$
f_{\alpha}(x)=\sum_{j=-m}^{m+1} \alpha_{j} \operatorname{dist}\left(x_{j}, x\right)^{2}
$$

$\left(\sum \alpha_{j}=1\right)$ with $x_{j} \in M$ has a unique minimum. This implies that the geodesic average is globally well-defined in Cartan-Hadamard manifolds.

Proof By Lemma 3, there exists a minimum of the function $f_{\alpha}$ and all its minima lie inside a compact ball. By the second part of Lemma 5, the function $f_{\alpha}$ is strictly convex, so the minimum is unique.

\section{Convergence result}

In this section, we prove that the Riemannian analogue of a linear subdivision scheme in a Cartan-Hadamard manifold converges for all input data, if the mask satisfies a contractivity condition with contractivity factor smaller than 1 , see Theorems 8 and 11. The condition implying convergence involves derived schemes (and iterates of derived schemes) and is analogous to a well-known criterion which applies in the linear case. This kind of result was previously only known for schemes with nonnegative mask (see [23, Theorem 5]). It has already been conjectured in [11].

\subsection{Contractivity condition}

We begin by adapting Lemma 3 of [23]. 
Lemma 7 Consider points $x_{j}$, coefficients $\alpha_{j}, \beta_{j}$, for $j=-m, \ldots, m+1$, and their centre of mass $x^{*}=\operatorname{av}(\alpha, x), x^{* *}=\operatorname{av}(\beta, x)$ in a Cartan-Hadamard manifold. Moreover, we assume that (3) holds. Then,

$$
\operatorname{dist}\left(x^{*}, x^{* *}\right) \leqslant\left(\sum_{j=-m}^{m+1}\left|\sum_{i \leqslant j} \alpha_{i}-\beta_{i}\right|\right) \cdot \max _{\ell} \operatorname{dist}\left(x_{\ell}, x_{\ell+1}\right) .
$$

The proof of this lemma is given in the Appendix.

Recall that a linear, binary subdivision scheme $S$ is given by $S x_{i}=\sum_{j \in \mathbb{Z}} a_{i-2 j} x_{j}$ with $\sum_{j \in \mathbb{Z}} a_{i-2 j}=1$ for all $i$. In order to obtain a convergence result for the Riemannian analogue $T$ of $S$, we have to estimate the distance between two consecutive points in the sequence $S^{k} x$. Let $\mu_{j}^{(r)}=\sum_{i \leqslant j} a_{r-2 i}$ and

$$
\mu=\max _{r \in\{1,2\}} \sum_{j=-m}^{m+1}\left|\mu_{j}^{(r+1)}-\mu_{j}^{(r)}\right| .
$$

Then, Lemma 7 implies that the subdivision rule $T$ obeys a so-called contractivity condition

$$
\operatorname{dist}\left(T^{k} x_{i+1}, T^{k} x_{i}\right) \leqslant \mu^{k} \cdot \sup _{\ell} \operatorname{dist}\left(x_{\ell}, x_{\ell+1}\right) .
$$

The factor $\mu$ is called contractivity factor. In Section 4.2, we show that the value of the contractivity factor $\mu$ in (10) is closely related to the norm of the derived scheme.

We make use of the result Hanne Hardering gave in [13, Lemma 2.3] again. In particular, she shows that all solutions of the minimisation problem stated in (7) lie inside a compact ball around $x_{0}$. The radius of this ball only depends on the chosen weights and the distances of $x_{i}, i=-m, \ldots, m$, from $x_{0}$. In our setting, this means that the points of the refined sequence are not too far from the initial sequence points. To be more precise, it follows that there exists a constant $C>0$ such that

$$
\operatorname{dist}\left(T x_{2 i}, x_{i}\right) \leqslant C \cdot \sup _{\ell} \operatorname{dist}\left(x_{\ell}, x_{\ell+1}\right), \quad i \in \mathbb{Z} .
$$

Subdivision schemes satisfying inequality (12) have been called displacement-safe by [8]. Together with (11), we conclude that

$$
\operatorname{dist}\left(T^{k+1} x_{2 i}, T^{k} x_{i}\right) \leqslant C \mu^{k} \varrho \text { with } \varrho:=\sup _{\ell} \operatorname{dist}\left(x_{\ell}, x_{\ell+1}\right) .
$$

In the linear case (see [5]), a contractivity factor smaller than 1 itself leads to a convergence result, but this condition is not sufficient in the nonlinear case. Here, we additionally need the fact that our schemes are displacement-safe as shown in [8] for manifold-valued subdivision schemes based on an averaging process. For interpolatory subdivision schemes however, a contractivity factor smaller than 1 entails convergence of the scheme since (12) is satisfied anyway, see [8, 20].

We now state our convergence result which generalises the result of [23].

Theorem 8 Consider a linear, binary, affine invariant subdivision scheme S. Denote by $T$ the Riemannian analogue of $S$ in a Cartan-Hadamard manifold $M$. Let $\mu$ be 
the contractivity factor defined by (10). If $\mu<1$, then $T$ converges to a continuous limit $T^{\infty} x$ for all input data $x$.

Proof Let $J=[a, b]$ be an interval and denote by $C(J, M)$ the continuous functions from $J$ to $M$. We use $c \mid J$ for the restriction of a map $c$ to an interval $J$. Denote by $c_{k}: \mathbb{R} \rightarrow M$ the broken geodesic which is the union of geodesic segments $c_{k} \mid\left[\frac{i}{2^{k}}, \frac{i+1}{2^{k}}\right]$ which connect successive points $T^{k} x_{i}$ and $T^{k} x_{i+1}$. We show that $\left(c_{k} \mid J\right)_{k \geqslant 0}$ is a Cauchy sequence in $C(J, M)$ for any $J$. The metric on $C(J, M)$ is given by $\operatorname{dist}(g, h):=\max _{t \in J} \operatorname{dist}(g(t), h(t))$. We now proceed as in the proof of Proposition 4 of [23]. Since $T$ satisfies (11) and is displacement-safe, it follows from the definition of the geodesics that

$$
\operatorname{dist}\left(c_{m}, c_{m+1}\right) \leqslant \varrho \mu^{m}+C \varrho \mu^{m}+\varrho \mu^{m+1} .
$$

Therefore,

$$
\operatorname{dist}\left(c_{m}, c_{n}\right) \leqslant(\varrho+C \varrho+\varrho \mu) \frac{\mu^{m}-\mu^{n}}{1-\mu}
$$

for all $m \leqslant n$. Thus, $\left(c_{k} \mid J\right)_{k \geqslant 0}$ is a Cauchy sequence in $C(J, M)$ for any interval $J=$ $[a, b]$. Completeness of the space $C(J, M)$ implies existence of the limit function $T^{\infty} x$.

Example 9 We compute the contractivity factor of the subdivision scheme introduced in Example 1. Using our previous results, we get

$$
\mu=\max \left\{\frac{28}{32}, \frac{8}{32}\right\}=\frac{28}{32}<1 \text {. }
$$

Thus, the Riemannian analogue of the linear scheme converges in Cartan-Hadamard manifolds for all input data. Figure 2 illustrates the action of this subdivision scheme in the hyperbolic plane.

Remark 1 So far, we considered subdivision schemes with dilation factor $N=2$. We note here that one can extend the convergence result given in Theorem 8 to subdivision schemes with arbitrary dilation factor. We still extend a linear subdivision
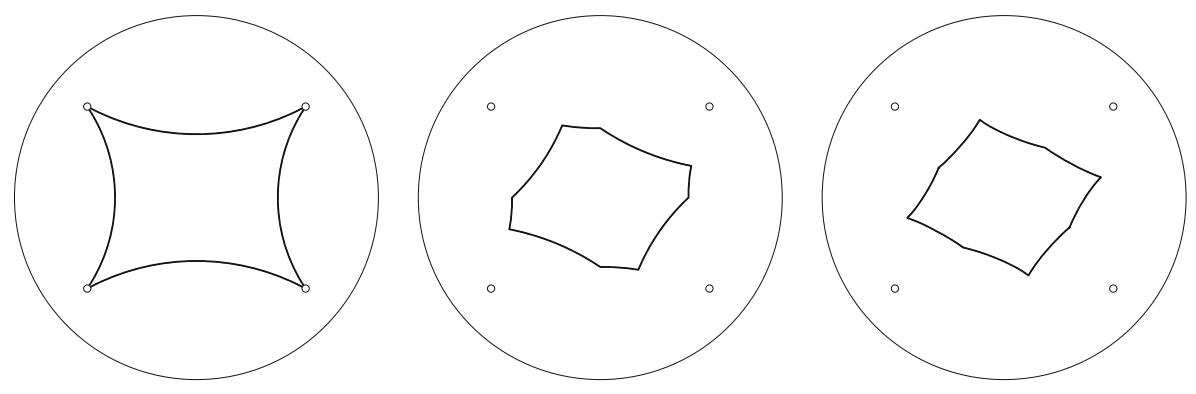

Fig. 2 Subdivision algorithm of Example 1 with initial data $x_{0}=(0.6,0.5), x_{1}=(0.6,-0.5), x_{2}=$ $(-0.6,-0.5)$ and $x_{3}=(-0.6,0.5)$ in the hyperbolic plane represented with the Poincare disk model. From left to right: initial polygon, polygon after one refinement step and polygon after four refinement steps 
scheme $S$ to its nonlinear counterpart $T$ by using the Riemannian analogue introduced in Section 2.2. Analogous to the binary case, we say that $T$ satisfies a contractivity condition with contractivity factor $\mu$ if

$$
\operatorname{dist}\left(T^{k} x_{i+1}, T^{k} x_{i}\right) \leqslant \mu^{k} \cdot \sup _{\ell} \operatorname{dist}\left(x_{\ell}, x_{\ell+1}\right), \quad i \in \mathbb{Z} .
$$

Also, we say that $T$ is displacement-safe if there exists a constant $C>0$ such that

$$
\operatorname{dist}\left((T x)_{N i}, x_{i}\right) \leqslant C \cdot \sup _{\ell} \operatorname{dist}\left(x_{\ell}, x_{\ell+1}\right), \quad i \in \mathbb{Z} .
$$

The convergence result now reads as follows.

Theorem 10 Let $T$ be the Riemannian analogue of the linear subdivision rule $S$ in a Cartan-Hadamard manifold M. Assume that (1) holds. Let $\mu_{j}^{(r)}=\sum_{i \leqslant j} a_{r-N i}$ and

$$
\mu=\max _{r \in\{1, \ldots, N\}} \sum_{j}\left|\mu_{j}^{(r+1)}-\mu_{j}^{(r)}\right| .
$$

If $\mu<1$, then $T$ converges to a continuous limit $T^{\infty} x$ for all input data $x$.

The convergence proof in the case $N>2$ is along the same lines as for $N=2$.

\subsection{Derived scheme}

For every linear, affine invariant subdivision scheme $S$, there exists the derived scheme $S^{*}$ given by the rule $S^{*} \Delta=N \Delta S$ with $\Delta x_{i}=x_{i+1}-x_{i}$, see, e.g. [11, Sec. 2.1]. In this section, we show that the contractivity factor (16) is closely related to the norm

$$
\left\|S^{*}\right\|:=\max _{r \in\{1, \ldots, N\}}\left\{\sum_{j}\left|a_{r-N j}^{*}\right|\right\}
$$

of the derived scheme $S^{*}$ with mask $a^{*}$. This result is not surprising since it holds in the linear case as well as for nonlinear subdivision schemes with nonnegative mask [23].

Theorem 11 Let $S$ be a linear, affine invariant subdivision rule with dilation factor $N$. Denote its derived scheme by $S^{*}$. If there exists an integer $m \geqslant 1$ such that $\frac{1}{N^{m}}\left\|S^{m *}\right\|<1$, then the Riemannian analogue of $S^{m}$ in a Cartan-Hadamard manifold converges for all input data.

We can reuse the proof of Theorem 5 of [23] to show Theorem 11. We repeat it here for the reader's convenience.

Proof Let $a^{*}=\left(a_{j}^{*}\right)_{j \in \mathbb{Z}}$ denote the mask of the derived scheme $S^{*}$. We consider the special input data $y=\left(y_{j}\right)_{j \in \mathbb{Z}}$ given by

$$
y_{j}=\left\{\begin{array}{c}
-1 \text { if } j \leqslant 0 \\
0 \text { else. }
\end{array}\right.
$$


We obtain

$$
\begin{aligned}
\frac{1}{N} a_{l}^{*} & =\frac{1}{N} \sum_{k} a_{l-N k}^{*}\left(y_{k+1}-y_{k}\right)=\frac{1}{N} S^{*}\left(y_{l+1}-y_{l}\right)=\frac{1}{N} S^{*} \Delta y_{l} \\
& =\Delta S y_{l}=S y_{l+1}-S y_{l}=\sum_{k \leqslant 0} a_{l-N k}-a_{l+1-N k}, \quad \text { and } \\
\frac{1}{N} a_{r-N j}^{*} & =\sum_{k \leqslant 0} a_{r-N(j+k)}-a_{r+1-N(j+k)}=\sum_{i \leqslant j} a_{r-N i}-a_{r+1-N i} .
\end{aligned}
$$

By (16), we get

$$
\sup _{r} \sum_{j}\left|\mu_{j}^{(r)}-\mu_{j}^{(r+1)}\right|=\frac{1}{N} \sup _{r} \sum_{j}\left|a_{r-N j}^{*}\right|=\frac{1}{N}\left\|S^{*}\right\| .
$$

Since the dilation factor of $S^{m}$ is $N^{m}$, Theorem 10 gives the desired result.

We have just seen that the contractivity factor (16) of the Riemannian analogue of a linear subdivision scheme $S$ is given by

$$
\mu=\frac{1}{N}\left\|S^{*}\right\|
$$

So in order to obtain a convergence result, it suffices to check if the norm of the derived scheme $S^{*}$ is smaller than the dilation factor. Even if this is not the case, we might get a convergence result by considering iterates of derived schemes $S^{m *}$, since the contractivity factor might decrease, see Section 7.1.

In [5], it is shown that if we ask for uniform convergence of a linear subdivision scheme $S$, the existence of an integer $m \geqslant 1$ such that $\frac{1}{N^{m}}\left\|S^{* m}\right\|<1$ is equivalent to the convergence of the scheme. Thus, Theorem 11 states that if the linear subdivision scheme converges uniformly, so does a certain Riemannian analogue of this scheme in Cartan-Hadamard manifolds.

\section{Hölder continuity}

It has been shown in [20] that the limit function of an interpolatory subdivision scheme for manifold-valued data has Hölder continuity $-\frac{\log \mu}{\log 2}$. Here, $\mu$ is a contractivity factor for the nonlinear analogue of the linear scheme. It depends only on the mask of the scheme. In [5], a similar inequality is proven for uniformly convergent subdivision schemes in linear spaces. We get the following related result.

Proposition 12 Let $T$ be the Riemannian analogue of a binary, affine invariant subdivision scheme $S$ which has contractivity factor $\mu<1$. Then, the limit curve $T^{\infty} x$ satisfies

$$
\operatorname{dist}\left(T^{\infty} x\left(t_{1}\right), T^{\infty} x\left(t_{2}\right)\right) \leqslant D\left|t_{2}-t_{1}\right|^{l},
$$

with

$$
D=2 \cdot\left(\frac{C \varrho+\varrho+\mu \varrho}{1-\mu}+\varrho\right) \quad \text { and } \quad \iota=1-\frac{\log \left\|S^{*}\right\|}{\log 2}
$$


for all $t_{1}, t_{2} \in \mathbb{R}$ with $\left|t_{1}-t_{2}\right|<1$ and all input data $x$, i.e. the limit curve is Hölder continuous with exponent $\iota$.

Here, the data-dependent constant $\varrho$ is defined by the maximal distance of successive data points which contribute to the limit curve in the interval under consideration.

The proof is contained in the Appendix.

Example 13 For our main Example 1 , we compute $\iota=-\log \left(\frac{28}{32}\right) / \log 2 \approx 0.1926$.

For subdivision schemes with arbitrary dilation factor, we obtain

Proposition 14 Let $T$ be the Riemannian analogue of a linear subdivision scheme $S$ in a Cartan-Hadamard manifold $M$ satisfying (1). Moreover, we assume that $T$ has contractivity factor $\mu<1$. Then, the limit curve $T^{\infty} x$ satisfies

$$
\operatorname{dist}\left(T^{\infty} x\left(t_{1}\right), T^{\infty} x\left(t_{2}\right)\right) \leqslant D\left|t_{2}-t_{1}\right|^{\iota}
$$

with

$$
D=2 \cdot \frac{C \varrho+\varrho+(N-1) \mu \varrho}{1-\mu}+N \varrho \quad \text { and } \quad \iota=1-\frac{\log \left\|S^{*}\right\|}{\log N}
$$

for all $t_{1}, t_{2} \in \mathbb{R}$ with $\left|t_{1}-t_{2}\right|<1$ and all input data $x$. Here, $N$ is the dilation factor and the data-dependent constant $\varrho$ is defined by the maximal distance of successive data points which contribute to the limit curve in the interval under consideration.

\section{The case of manifolds which are not simply connected}

We explain how to extend our previous results to a complete Riemannian manifold $M$ with sectional curvature $K \leqslant 0$, i.e. we drop the assumption of simple connectedness. We use the fact that $M$ has a so-called simply connected covering (universal covering) $\tilde{M}$. This is a simply connected manifold which projects onto $M$ in a locally diffeomorphic way. The Riemannian metric on $M$ is transported to $\tilde{M}$ by declaring the projection $\pi: \tilde{M} \rightarrow M$ a local isometry. An example is shown by Fig. 3, where a strip of infinite length and width 1 wraps around the cylinder of height 1 infinitely many times. For the general theory of coverings, see, e.g. [14]. Each data point $x_{j}$ in $M$ has a potentially large number of preimages $\pi^{-1}\left(x_{j}\right)$.

\subsection{Re-definition of the Riemannian analogue of a linear scheme}

So far our initial data always consisted of a sequence of points in $M$. Now we additionally choose a path $c(t)$ which connects the data points $x_{j}$ in the correct order: we have $c\left(t_{j}\right)=x_{j}$ for suitable parameter values $\ldots<t_{j}<t_{j+1}<\ldots$ Such a path is 
not unique, see Fig. 3. By well-known properties of the simply connected covering, this path can be uniquely lifted to a path $\tilde{c}(t)$ in $\tilde{M}$ which projects onto the original path $c(t)$, once a preimage $\tilde{x}_{0}$ with $\pi\left(\tilde{x}_{0}\right)=x_{0}$ has been chosen. This means that for all indices $j$, we have

$$
\tilde{c}\left(t_{j}\right)=\tilde{x}_{j}, \quad \text { with } \pi\left(\tilde{x}_{j}\right)=x_{j} .
$$

We can now simply apply the Riemannian analogue $\tilde{T}$ of the linear scheme $S$ which operates on data from $\tilde{M}$, because $\tilde{M}$ is Cartan-Hadamard by construction. Note that there is no Riemannian analogue of $S$ in $M$, since $M$ is not simply connected and geodesic averages are not well-defined in general. However, if our input data is a sequence $x_{j}$ together with a connecting path as described above, we may let

$$
T x=\pi(\tilde{T} \tilde{x}) \quad \text { where } \tilde{x} \text { arises from } x \text { by lifting. }
$$

We can still call $T$ a natural Riemannian analogue of the linear subdivision scheme $S$.

Lemma 15 For any given input data $\left(x_{j}\right)$, the refined data $(T x)_{j}$ computed by the Riemannian analogue $T$ of a linear subdivision scheme $S$ depends only on the homotopy class of the path $c(t)$ which is used to connect the data points.

Proof First we show that $T x$ does not depend on the choice of the preimage $\tilde{x}_{0}$ in the covering space $\tilde{M}$ : if another preimage $\tilde{x}_{0}^{\prime}$ is chosen, there is an isometric
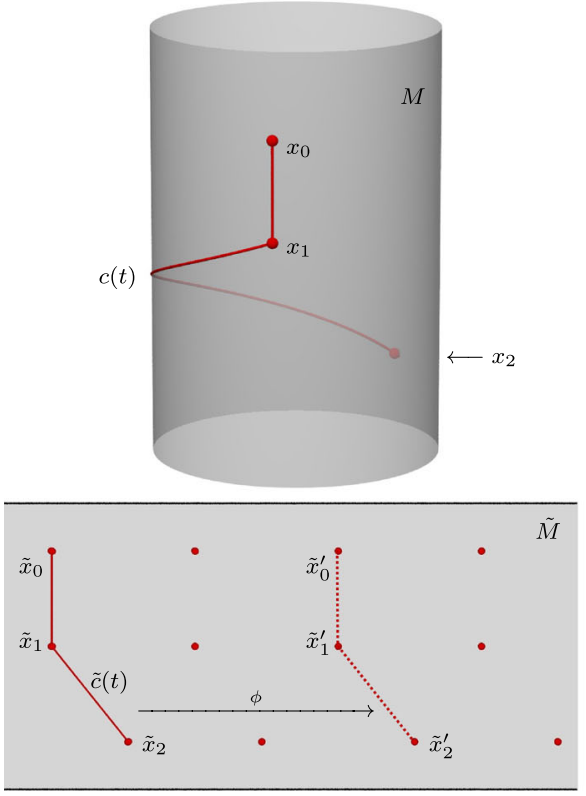
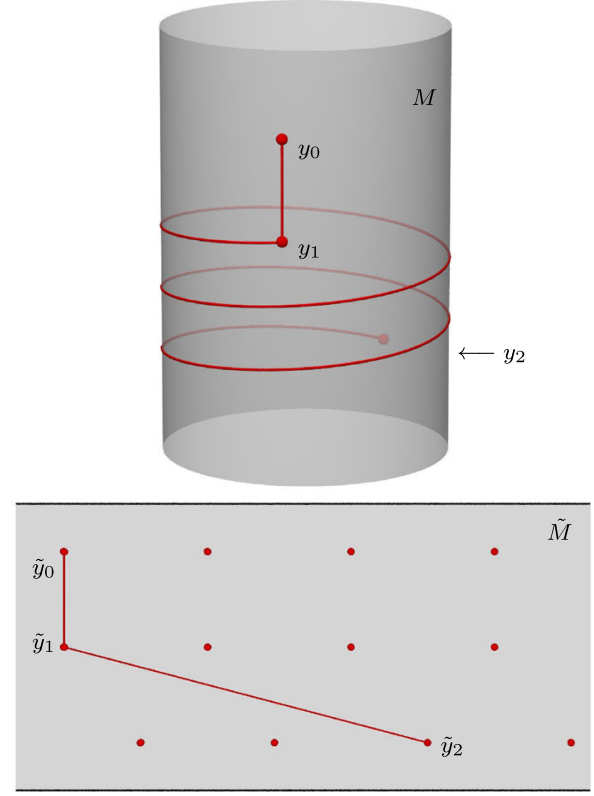

Fig. 3 Top: initial data on a cylinder $M=S^{1} \times[0,1]$ together with connecting paths. Bottom: their lift to the universal covering $\tilde{M}$, which is the strip $(-\infty, \infty) \times[0,1]$. The various possible liftings are mapped onto each other by a deck transformation $\phi$ 
deck transformation $\phi: \tilde{M} \rightarrow \tilde{M}$ which maps the original lifting to the new one and which commutes with the covering projection $\pi$. The action of $\tilde{T}$ is invariant under isometries, so $\pi\left(\tilde{T} \tilde{x}^{\prime}\right)=\pi(\tilde{T} \phi(\tilde{x}))=\pi(\phi(\tilde{T} \tilde{x}))=\pi(\tilde{T} \tilde{x})$. Further, it is well known that the lifted location $\tilde{x}_{j}$ of any individual data point $x_{j}$ depends only on the homotopy class of the path $c$, cf. [14].

With this modification of the notion of input data, our main result Theorem 11 now reads as follows:

Theorem 16 Let $M$ be a complete manifold with $K \leqslant 0$, and let $S$ be a linear, affine invariant subdivision rule with dilation factor $N$. Denote by $S^{*}$ its derived scheme. If there exists an integer $m \geqslant 1$ such that $\frac{1}{N^{m}}\left\|S^{m *}\right\|<1$, then the Riemannian analogue of $S^{m}$ in $M$ produces continuous limits for all input data.

\section{Examples}

\subsection{Four-point scheme}

Consider the general four-point scheme $S$ introduced in (5). We would like to know for which values of $\omega \in(0, \infty)$ the Riemannian analogue $T$ of $S$ converges. The mask of the derived scheme is given by $a_{-2}^{*}=a_{3}^{*}=-2 \omega, a_{-1}^{*}=a_{2}^{*}=2 \omega$ and $a_{0}^{*}=a_{1}^{*}=1$. Thus by Theorem 8 , the contractivity factor is $\mu=2|\omega|+\frac{1}{2}$ and $T$ converges for arbitrary input data if $-\frac{1}{4}<\omega<\frac{1}{4}$. For $-\frac{1}{2}<\omega \leqslant 0$, this has already been known $[9,10]$. In this case, the mask is nonnegative.

In particular, we obtain a contractivity factor of $\mu=\frac{5}{8}$ for the well-studied case of the four-point scheme with $\omega=\frac{1}{16}$. By Proposition 12, we obtain a Hölder exponent of $\iota \approx 0.6781$. Figure 4 shows an example of the four-point scheme in the hyperbolic plane for $\omega=0.23$.

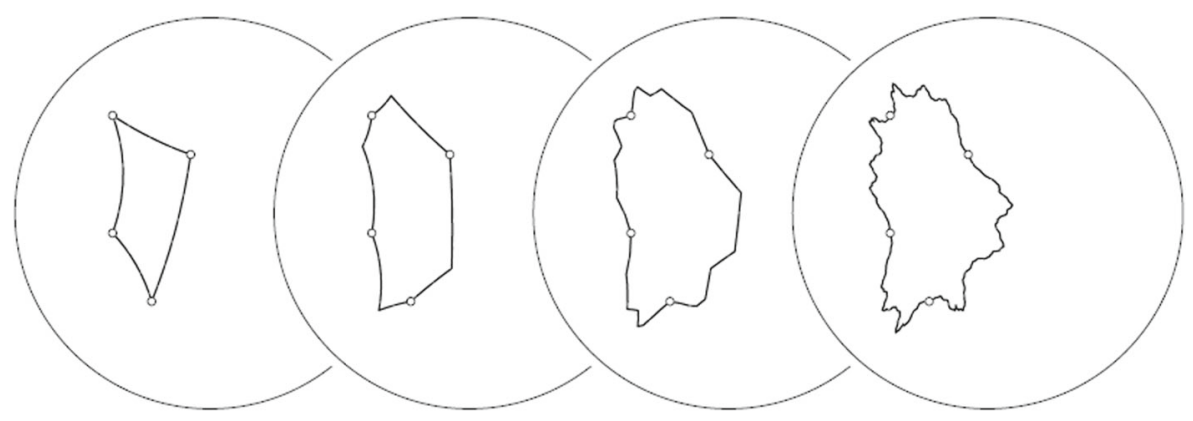

Fig. 4 The four-point scheme with $\omega=0.23$ in the hyperbolic plane. Left to right: initial polygon, polygon after one refinement step, polygon after three refinement steps and limit curve. The limit curve is Hölder continuous with exponent 0.06 
Now we consider two rounds of the four-point scheme as one round of a subdivision scheme with dilation factor $N=4$ which for simplicity is again called $S$. If $\omega=\frac{1}{16}$, our refinement rule is then given by

$$
\begin{aligned}
(S x)_{4 i} & =x_{i}, \\
(S x)_{4 i+1} & =\frac{1}{16^{2}}\left(x_{i-2}-18 x_{i-1}+216 x_{i}+66 x_{i+1}-9 x_{i+2}\right), \\
(S x)_{4 i+2} & =\frac{1}{16^{2}}\left(-16 x_{i-1}+144 x_{i}+144 x_{i+1}-16 x_{i+2}\right), \\
(S x)_{4 i+3} & =\frac{1}{16^{2}}\left(-9 x_{i-1}+66 x_{i}+216 x_{i+1}-18 x_{i+2}+x_{i+3}\right) .
\end{aligned}
$$

The contractivity factor is

$$
\mu=\max \left\{\frac{84}{16^{2}}, \frac{80}{16^{2}}\right\}=\frac{84}{16^{2}} \approx 0.3281 .
$$

Theorem 10 again confirms that a Riemannian analogue converges to a continuous limit function for all input data. Proposition 14 yields a Hölder exponent of $\iota \approx$ 0.8039 .

Acknowledgments Open access funding provided by Austrian Science Fund (FWF).

Funding information The authors acknowledge the support of the Austrian Science Fund (FWF): This research was supported by the doctoral programme Discrete Mathematics (grant no. W1230) and by the SFB-Transregio programme Discretization in geometry and dynamics (grant no. I705).

Open Access This article is distributed under the terms of the Creative Commons Attribution 4.0 International License (http://creativecommons.org/licenses/by/4.0/), which permits unrestricted use, distribution, and reproduction in any medium, provided you give appropriate credit to the original author(s) and the source, provide a link to the Creative Commons license, and indicate if changes were made.

\section{Appendix}

We give the proofs of the two Lemmas 5 and 7 which use the concept of Jacobi fields. Therefore, we first briefly recall their definition and properties. Finally, we prove Proposition 12.

\section{A.1 Jacobi fields}

Denote by $c(u, s)$ a one-parameter family of geodesics parametrised by $s$, with $u$ being the parameter along each geodesic. Let $c^{\prime}(u, s):=\frac{d}{d u} c(u, s)$. Then for fixed $s=s_{0}, J(u)=c^{\prime}\left(u, s_{0}\right)$ is a Jacobi vector field along the geodesic $c\left(\cdot, s_{0}\right)$. It is known that Jacobi fields are solutions of the linear second-order differential equation $J^{\prime \prime}+R\left(c^{\prime}, J\right) c^{\prime}=0$, with $R$ denoting the Riemann curvature tensor. For any given geodesic, there is a linear space of Jacobi vector fields whose dimension is $2 \operatorname{dim} M$. Jacobi fields are an important tool in global Riemannian geometry because on the one hand, geodesics cannot be shortest curves if they admit Jacobi fields with two zeros, 
and on the other hand, the behaviour of Jacobi fields is guided by $R$. For this reason, Jacobi fields are a well-investigated topic. We refer to the textbook of do Carmo [1] for a general introduction.

\section{A.2 Proofs}

Proof of Lemma 5 Recall the definition of $f_{\alpha}$ by (9). Let $\gamma:[0,1] \rightarrow M$ be a geodesic and

$$
c_{t}(u, s)=\exp _{x_{\sigma(t)}}\left(u \cdot \exp _{x_{\sigma(t)}}^{-1} \gamma(s)\right)
$$

For any $s$, the geodesic $c_{t}(\cdot, s)$ connects $x_{\sigma(t)}$ with $\gamma(s)$. Those geodesics exist and are unique since $M$ is Cartan-Hadamard. Additionally, let $c_{t}^{\prime}(u, s):=\frac{d}{d u} c_{t}(u, s)$ and $\dot{c}_{t}(u, s):=\frac{d}{d s} c_{t}(u, s)$. By construction, dist $\left(x_{\sigma(t)}, \gamma(s)\right)=\left\|c_{t}^{\prime}(u, s)\right\|$. For each $t, s$ the vector field $J(u)=\dot{c}_{t}(u, s)$ along the geodesic $u \mapsto c_{t}(u, s)$ is a Jacobi field. Since

$$
\begin{aligned}
f_{\alpha}(\gamma(s)) & =\left(-\int_{0}^{\alpha_{-}}+\int_{\alpha_{-}}^{\alpha_{-}+\alpha_{+}}\right) \operatorname{dist}\left(x_{\sigma(t)}, \gamma(s)\right)^{2} d t \\
& =\left(-\int_{0}^{\alpha_{-}}+\int_{\alpha_{-}}^{\alpha_{-}+\alpha_{+}}\right)\left\langle c_{t}^{\prime}(u, s), c_{t}^{\prime}(u, s)\right\rangle d t
\end{aligned}
$$

we obtain

$$
\frac{1}{2} \frac{d}{d s} f_{\alpha}(\gamma(s))=\left(-\int_{0}^{\alpha_{-}}+\int_{\alpha_{-}}^{\alpha_{-}+\alpha_{+}}\right)\left\langle\frac{\nabla}{\partial s} c_{t}^{\prime}(u, s), c_{t}^{\prime}(u, s)\right\rangle d t .
$$

Here, $\frac{\nabla}{\partial s}$ denotes the covariant derivative along the curve $\gamma(s)$. In the following, we use the facts that $\left\|c_{t}^{\prime}(u, s)\right\|$ does not depend on $s, \frac{\nabla}{\partial s} c_{t}^{\prime}(u, s)=\frac{\nabla}{\partial u} \dot{c}_{t}(u, s)$ and finally that $\frac{\nabla}{\partial u} c_{t}^{\prime}(u, s)=0$ since $c$ is a geodesic. This leads to

$$
\begin{aligned}
& \left(-\int_{0}^{\alpha_{-}}+\int_{\alpha_{-}}^{\alpha_{-}+\alpha_{+}}\right)\left\langle\frac{\nabla}{\partial s} c_{t}^{\prime}(u, s), c_{t}^{\prime}(u, s)\right\rangle d t \\
= & \left(-\int_{0}^{\alpha_{-}}+\int_{\alpha_{-}}^{\alpha_{-}+\alpha_{+}}\right) \int_{0}^{1}\left\langle\frac{\nabla}{\partial s} c_{t}^{\prime}(u, s), c_{t}^{\prime}(u, s)\right\rangle d u d t \\
= & \left(-\int_{0}^{\alpha_{-}}+\int_{\alpha_{-}}^{\alpha_{-}+\alpha_{+}}\right) \int_{0}^{1}\left\langle\frac{\nabla}{\partial u} \dot{c}_{t}(u, s), c_{t}^{\prime}(u, s)\right\rangle d u d t \\
= & \left(-\int_{0}^{\alpha_{-}}+\int_{\alpha_{-}}^{\alpha_{-}+\alpha_{+}}\right) \int_{0}^{1} \frac{d}{d u}\left\langle\dot{c}_{t}(u, s), c_{t}^{\prime}(u, s)\right\rangle d u d t .
\end{aligned}
$$

Since $\dot{c}_{t}(0, s)=0$, we finally obtain

$$
\frac{1}{2} \frac{d}{d s} f_{\alpha}(\gamma(s))=\left(-\int_{0}^{\alpha_{-}}+\int_{\alpha_{-}}^{\alpha_{-}+\alpha_{+}}\right)\left\langle\dot{c}_{t}(1, s), c_{t}^{\prime}(1, s)\right\rangle .
$$


Observe that $c_{t}^{\prime}(1, s)=-\exp _{\gamma(s)}^{-1} x_{\sigma(t)}$ (by definition of the exponential map) and $\dot{c}_{t}(1, s)=\dot{\gamma}(s)$ (by construction) are independent of $t$. Therefore,

$$
\frac{1}{2} \frac{d}{d s} f_{\alpha}(\gamma(s))=\left\langle\dot{\gamma}(s),\left(\int_{0}^{\alpha_{-}}-\int_{\alpha_{-}}^{\alpha_{-}+\alpha_{+}}\right) \exp _{\gamma(s)}^{-1} x_{\sigma(t)} d t\right\rangle .
$$

By the definition of the gradient, we conclude that

$$
\frac{1}{2} \operatorname{grad} f_{\alpha}(x)=\left(\int_{0}^{\alpha_{-}}-\int_{\alpha_{-}}^{\alpha_{-}+\alpha_{+}}\right) \exp _{x}^{-1} x_{\sigma(t)} d t .
$$

Using (8), we see that

$$
\begin{aligned}
\frac{1}{2} \frac{d^{2}}{d s^{2}} f_{\alpha}(\gamma(s)) & =\left(-\int_{0}^{\alpha_{-}}+\int_{\alpha_{-}}^{\alpha_{-}+\alpha_{+}}\right)\left\langle\dot{c}_{t}(1, s), \frac{\nabla}{\partial u} \dot{c}_{t}(1, s)\right\rangle d t \\
& =\left(-\int_{0}^{\alpha_{-}}+\int_{\alpha_{-}}^{\alpha_{-}+\alpha_{+}}\right)\left\langle J(1), J^{\prime}(1)\right\rangle d t \\
& =\left\langle J(1), J^{\prime}(1)\right\rangle \geqslant\langle\dot{\gamma}(s), \dot{\gamma}(s)\rangle .
\end{aligned}
$$

To obtain the inequality above, we used the following relations between the Jacobi field and its derivative

$$
J^{\prime}(1)^{\tan }=J(1)^{\tan } \quad \text { and } \quad\left\langle J^{\prime}(1)^{\text {norm }}, J(1)\right\rangle \geqslant\left\langle J(1)^{\text {norm }}, J(1)\right\rangle,
$$

where $J^{\tan }$ (resp. $J^{\text {norm }}$ ) denotes the tangential (resp. normal) part of the Jacobi field; see Appendix A in [15] for more details. Here, we used the fact that the sectional curvature of $M$ is bounded above by zero.

Remark 2 We note that a direct further differentiation of (17) yields

$$
\frac{d}{d s}\left(\frac{1}{2} \frac{d}{d s} f_{\alpha}(\gamma(s))\right)=\left(-\int_{0}^{\alpha_{-}}+\int_{\alpha_{-}}^{\alpha_{-}+\alpha_{+}}\right)\left\langle\dot{c}_{t}(1, s), \frac{\nabla}{\partial u} \frac{d}{d s} c_{t}(1, s)\right\rangle .
$$

Thus,

$$
\frac{\nabla}{\partial s} \frac{1}{2} \operatorname{grad} f_{\alpha}(\gamma(s))=\left(\int_{0}^{\alpha_{-}}-\int_{\alpha_{-}}^{\alpha_{-}+\alpha_{+}}\right) \frac{\nabla}{\partial u} \frac{d}{d s} c_{t}(1, s) d t .
$$

This equality is used in the next proof.

To prove the next result, we make use of the representation of $f_{\alpha}$ (resp. $f_{\beta}$ ) as in (9) in terms of the function $\sigma$ (resp. $\tau$ ). Before we give the proof of Lemma 7, we illustrate the idea by means of our main example:

Example 17 From Example 4, we know that

$$
f_{\alpha}(x)=-\int_{0}^{\alpha_{-}} \operatorname{dist}\left(x_{\sigma(t)}, x\right)^{2} d t+\int_{\alpha_{-}}^{\alpha_{-}+\alpha_{+}} \operatorname{dist}\left(x_{\sigma(t)}, x\right)^{2} d t .
$$

Similarly, we obtain

$$
f_{\beta}(x)=-\int_{0}^{\beta_{-}} \operatorname{dist}\left(x_{\tau(t)}, x\right)^{2} d t+\int_{\beta_{-}}^{\beta_{-}+\beta_{+}} \operatorname{dist}\left(x_{\tau(t)}, x\right)^{2} d t,
$$


with $\beta_{-}=\frac{2}{32}, \beta_{+}=\frac{34}{32}$ and

$$
\tau(t)=\left\{\begin{aligned}
-1 \text { for } t & \in\left[0, \frac{1}{32}\right] \\
2 \text { for } t & \in\left(\frac{1}{32}, \frac{2}{32}\right] \\
0 \text { for } t & \in\left(\frac{2}{32}, \frac{15}{32}\right] \\
1 \text { for } t & \in\left(\frac{15}{32}, \frac{36}{32}\right] .
\end{aligned}\right.
$$

In order to get the desired result in Lemma 7, we estimate the distance between the gradients of the functions $f_{\alpha}$ and $f_{\beta}$ at the point $x^{*}=\operatorname{av}(\alpha, x)$ (as explained in more detail in the proof of the Lemma 7). To be able to do so, we make use of Lemma 5 and convert the resulting four integrals into two. In this case, we get

$$
\begin{aligned}
& \left\|\frac{1}{2} \operatorname{grad} f_{\beta}\left(x^{*}\right)-\frac{1}{2} \operatorname{grad} f_{\alpha}\left(x^{*}\right)\right\|=\left\|\sum_{j=-1}^{2}\left(\alpha_{j}-\beta_{j}\right) \exp _{x *}^{-1} x_{j}\right\| \\
= & \left\|-\int_{0}^{\frac{8}{32}} \exp _{x *}^{-1} x_{v\left(t+\frac{8}{32}\right)} d t+\int_{0}^{\frac{8}{32}} \exp _{x *}^{-1} x_{v(t)} d t\right\|,
\end{aligned}
$$

with

$$
v(t)=\left\{\begin{array}{l}
0 \text { for } t \in\left[0, \frac{8}{32}\right] \\
1 \text { for } t \in\left(\frac{8}{32}, \frac{16}{32}\right] .
\end{array}\right.
$$

Note that the construction of the function $v$ is similar to the one of $\sigma$ in (9).

We are now ready to give the proof of Lemma 7 which follows the structure in [23] and the ideas of [15].

Proof of Lemma 7 To obtain a lower bound for the absolute value of the gradient of $\frac{1}{2} f_{\alpha}(x)$, we make use of Theorem 1.5. in [15]. Let $\gamma$ be the geodesic starting from $x^{*}$ and ending in $x$ and let $c_{t}(u, s)=\exp _{x_{\sigma(t)}}\left(u \cdot \exp _{x_{\sigma(t)}}^{-1} \gamma(s)\right)$ be the family of geodesics from $x_{\sigma(t)}$ to $\gamma(s)$. We apply the Cauchy-Schwarz inequality and the fact that $\operatorname{grad} f_{\alpha}\left(x^{*}\right)=0$ by definition of $x^{*}$ to obtain

$$
\left\|\frac{1}{2} \operatorname{grad} f_{\alpha}(\gamma(1))\right\| \cdot\|\dot{\gamma}(1)\| \geqslant \int_{0}^{1} \frac{d}{d s}\left\langle\frac{1}{2} \operatorname{grad} f_{\alpha}(\gamma(s)), \dot{\gamma}(s)\right\rangle d s .
$$

By Remark 2, we conclude

$$
\begin{aligned}
& \left\|\frac{1}{2} \operatorname{grad} f_{\alpha}(\gamma(1))\right\| \cdot\|\dot{\gamma}(1)\| \\
& \quad \geqslant\left(-\int_{0}^{\alpha_{-}}+\int_{\alpha_{-}}^{\alpha_{-}+\alpha_{+}}\right) \int_{0}^{1}\left\langle\frac{\nabla}{\partial u} \frac{d}{d s} c_{t}(1, s), \dot{c}_{t}(1, s)\right\rangle d s d t,
\end{aligned}
$$

with $\dot{c}_{t}(u, s)=\frac{d}{d s} c_{t}(u, s)$. As in the proof of Lemma 5, let $J(u)=\dot{c}_{t}(u, s)$ denote the Jacobi field along the curve $u \mapsto c_{t}(u, s)$. The dependence on $s$ and $t$ is not 
indicated in the notation. We have $J(1)=\dot{\gamma}(s)$ and $J^{\prime}(1)=\frac{\nabla}{\partial u} \dot{c}_{t}(1, s)$. Using (8), we obtain

$$
\begin{aligned}
\left\|\frac{1}{2} \operatorname{grad} f_{\alpha}(\gamma(1))\right\| \cdot\|\dot{\gamma}(1)\| & \geqslant\left(-\int_{0}^{\alpha_{-}}+\int_{\alpha_{-}}^{\alpha_{-}+\alpha_{+}}\right) \int_{0}^{1}\left\langle J^{\prime}(1), J(1)\right\rangle d s d t \\
& =\left\langle J^{\prime}(1), J(1)\right\rangle \geqslant\langle\dot{\gamma}(s), \dot{\gamma}(s)\rangle .
\end{aligned}
$$

The last inequality follows in the same way as in the proof of Lemma 5. By the definition of the geodesic $\gamma$, we have $\|\dot{\gamma}(s)\|=\operatorname{dist}\left(x, x^{*}\right)$ and conclude that

$$
\left\|\frac{1}{2} \operatorname{grad} f_{\alpha}(x)\right\| \geqslant \operatorname{dist}\left(x, x^{*}\right) \text {. }
$$

By definition of $x^{*}$, we have $\operatorname{grad} f_{\alpha}\left(x^{*}\right)=0$. Together with Lemma 5, we obtain

$$
\left\|\frac{1}{2} \operatorname{grad} f_{\beta}\left(x^{*}\right)\right\|=\left\|\frac{1}{2} \operatorname{grad} f_{\beta}\left(x^{*}\right)-\frac{1}{2} \operatorname{grad} f_{\alpha}\left(x^{*}\right)\right\|=\left\|\sum_{j=-m}^{m+1}\left(\alpha_{j}-\beta_{j}\right) \exp _{x *}^{-1} x_{j}\right\| .
$$

We define the sequence $\delta=\left(\delta_{j}\right)_{j=-m, \ldots, m+1}$ by $\delta_{j}=\alpha_{j}-\beta_{j}$. Let $v$ be the function constructed as $\sigma$ in (9) with respect to the coefficients $\delta$, i.e. the value of $v$ is constant in intervals of length $\left|\delta_{j}\right|$ and given by the corresponding index. Denote by $\delta_{-}$(resp. $\delta_{+}$) the sum of the absolute values of the negative (resp. nonnegative) coefficients of $\delta$. Equation (3) implies that $\delta_{-}=\delta_{+}$. As in (9), we rewrite the sum above as an integral

$$
\begin{aligned}
& \left\|\sum_{j=-m}^{m+1}\left(\alpha_{j}-\beta_{j}\right) \exp _{x *}^{-1} x_{j}\right\|=\left\|-\int_{0}^{\delta_{-}} \exp _{x *}^{-1} x_{v(t)} d t+\int_{\delta_{-}}^{\delta_{-}+\delta_{+}} \exp _{x *}^{-1} x_{v(t)} d t\right\| \\
= & \left\|\int_{0}^{\delta_{-}}\left(-\exp _{x *}^{-1} x_{v(t)}+\exp _{x *}^{-1} x_{v\left(t+\delta_{-}\right)}\right) d t\right\| \\
\leqslant & \int_{0}^{\delta_{-}}\left\|\exp _{x *}^{-1} x_{v\left(t+\delta_{-}\right)}-\exp _{x *}^{-1} x_{v(t)}\right\| d t .
\end{aligned}
$$

With the help of (19), we conclude that

$$
\begin{aligned}
\operatorname{dist}\left(x^{*}, x^{* *}\right) & \leqslant\left\|\frac{1}{2} \operatorname{grad} f_{\beta}\left(x^{*}\right)\right\| \leqslant \int_{0}^{\delta_{-}}\left\|\exp _{x *}^{-1} x_{v\left(t+\delta_{-}\right)}-\exp _{x *}^{-1} x_{v(t)}\right\| d t \\
& \leqslant \int_{0}^{\delta_{-}} \operatorname{dist}\left(x_{v\left(t+\delta_{-}\right)}, x_{v(t)}\right) d t \\
& \leqslant \int_{0}^{\delta_{-}}\left|v\left(t+\delta_{-}\right)-v(t)\right| d t \cdot \max _{\ell} \operatorname{dist}\left(x_{\ell}, x_{\ell+1}\right) .
\end{aligned}
$$

To obtain the third inequality above, we used the fact that in Cartan-Hadamard manifolds, the exponential map does not decrease distances, see for example [16].

It remains to show that

$$
\int_{0}^{\delta_{-}}\left|v\left(t+\delta_{-}\right)-v(t)\right| d t=\sum_{j=-m}^{m+1}\left|\sum_{i \leqslant j} \alpha_{i}-\beta_{i}\right|
$$


To do that, we split the sequence of coefficients $\delta$ in two sequences $\eta^{1}, \eta^{2}$ defined by

$$
\eta_{j}^{1}:=\left\{\begin{array}{ll}
\delta_{j} & \text { if } \delta_{j} \geqslant 0 \\
0 & \text { else }
\end{array} \quad \text { and } \quad \eta_{j}^{2}:= \begin{cases}\left|\delta_{j}\right| & \text { if } \delta_{j}<0 \\
0 & \text { else. }\end{cases}\right.
$$

Similarly to the construction in the proof of Lemma 3 of [23], we consider the function $\epsilon_{1}$ given by

$$
\epsilon_{1}:\left[0, \delta_{-}\right] \rightarrow\{-m, \ldots, m+1\}, \quad \epsilon_{1}(t):=\sup \left\{j \mid \sum_{i \leqslant j} \eta_{i}^{1}<t\right\}+1 .
$$

Analogously, we define $\epsilon_{2}$ for the sequence $\eta^{2}$. We finally obtain

$$
\begin{aligned}
& \int_{0}^{\delta_{-}}\left|v\left(t+\delta_{-}\right)-v(t)\right| d t=\int_{0}^{\delta_{-}}\left|\epsilon_{1}(t)-\epsilon_{2}(t)\right| d t \\
= & \sum_{j=-m}^{m+1}\left|\sum_{i \leqslant j} \eta_{i}^{1}-\sum_{i \leqslant j} \eta_{i}^{2}\right|=\sum_{j=-m}^{m+1}\left|\sum_{i \leqslant j} \alpha_{i}-\beta_{i}\right| .
\end{aligned}
$$

This concludes the proof of Lemma 7.

Finally, we give the proof of Proposition 12.

Proof of Proposition 12 Assume that $t_{1}, t_{2} \in \mathbb{R}$ with $\left|t_{1}-t_{2}\right|<1$. Then, there exists an integer $k \in \mathbb{Z}$ such that $2^{-k-1} \leqslant\left|t_{2}-t_{1}\right| \leqslant 2^{-k}$. As in the proof of Theorem 8 , let $c_{k}$ be the union of geodesic segments $c_{k} \mid\left[\frac{i}{2^{k}}, \frac{i+1}{2^{k}}\right]$ connecting the points $T^{k} x_{i}$ and $T^{k} x_{i+1}$. Together with (11), we obtain

$$
\operatorname{dist}\left(c_{k+1}\left(t_{1}\right), c_{k+1}\left(t_{2}\right)\right) \leqslant 2 \sup _{\ell} \operatorname{dist}\left(T^{k+1} x_{\ell+1}, T^{k+1} x_{\ell}\right) \leqslant 2 \mu^{k+1} \varrho .
$$

Using (14), we have

$$
\begin{aligned}
& \operatorname{dist}\left(T^{\infty} x(t), c_{k+1}(t)\right) \leqslant \lim _{\ell \rightarrow \infty} \operatorname{dist}\left(c_{\ell}(t), c_{k+1}(t)\right) \\
& \leqslant \sum_{j=k+1}^{\infty} \operatorname{dist}\left(c_{j}(t), c_{j+1}(t)\right)=\frac{C \varrho+\varrho+\mu \varrho}{1-\mu} \mu^{k+1}
\end{aligned}
$$

for all $t \in \mathbb{R}$. Summarising the previous two observations leads to

$$
\begin{aligned}
& \operatorname{dist}\left(T^{\infty} x\left(t_{1}\right), T^{\infty} x\left(t_{2}\right)\right) \\
\leqslant & \operatorname{dist}\left(T^{\infty} x\left(t_{1}\right), c_{k+1}\left(t_{1}\right)\right)+\operatorname{dist}\left(c_{k+1}\left(t_{1}\right), c_{k+1}\left(t_{2}\right)\right)+\operatorname{dist}\left(c_{k+1}\left(t_{2}\right), T^{\infty} x\left(t_{2}\right)\right) \\
\leqslant & D \mu^{k+1} .
\end{aligned}
$$

Since $\left|t_{2}-t_{1}\right| \leqslant 2^{-k}$, taking the logarithm shows that $\mu^{k+1} \leqslant \mu^{-\log _{2}\left(\left|t_{2}-t_{1}\right|\right)}$. We conclude that

$$
\operatorname{dist}\left(T^{\infty} x\left(t_{1}\right), T^{\infty} x\left(t_{2}\right)\right) \leqslant D\left(2^{\log _{2}\left(\left|t_{2}-t_{1}\right|\right)}\right)^{-\log _{2}(\mu)} \leqslant D\left|t_{2}-t_{1}\right|^{\iota},
$$

with $\iota=-\frac{\log \mu}{\log 2}=1-\frac{\log \left\|S^{*}\right\|}{\log 2}$. Here, the last equality holds because $\mu=\frac{1}{2}\left\|S^{*}\right\|$. 


\section{References}

1. do Carmo, M.P.: Riemannian Geometry. Birkhäuser (1992)

2. Cavaretta, A.S., Dahmen, W., Michelli, C.A.: Stationary subdivision. Memoirs Am. Math. Soc., 93 (1991)

3. Chaikin, G.M.: An algorithm for high speed curve generation. Comput. Graphics Image Process. 3, 346-349 (1974)

4. Donoho, D.: Wavelet-type representation of Lie-valued data. talk at the IMI "Approximation and Computation" meeting, May 12-17, Charleston SC (2001)

5. Dyn, N.: Subdivision schemes in CAGD. In: Light, W.A. (ed.) Advances in Numerical Analysis, vol. II, pp. 36-104. Oxford Univ. Press (1992)

6. Dyn, N., Gregory, J., Levin, D.: A 4-point interpolatory subdivision scheme for curve design. Comput. Aided Geom. Des. 4, 257-268 (1987)

7. Dyn, N., Sharon, N.: A global approach to the refinement of manifold data. Math. Comput. 86(303), 375-395 (2017)

8. Dyn, N., Sharon, N.: Manifold-valued subdivision schemes based on geodesic inductive averaging. J. Comput. Appl. Math. 311, 54-67 (2017)

9. Ebner, O.: Convergence of iterative schemes in metric spaces. Proc. Am. Math. Soc. 141, 677-686 (2013)

10. Ebner, O.: Stochastic aspects of refinement schemes on metric spaces. SIAM J. Numer. Anal. 52, 717-734 (2014)

11. Grohs, P.: A general proximity analysis of nonlinear subdivision schemes. SIAM J. Math. Anal. 42, 729-750 (2010)

12. Grohs, P., Wallner, J.: Log-exponential analogues of univariate subdivision schemes in Lie groups and their smoothness properties. In: Neamtu, M., Schumaker, L.L. (eds.) Approximation Theory XII: San Antonio 2007, pp. 181-190. Nashboro Press (2008)

13. Hardering, H.: Intrinsic Discretization Error Bounds for Geodesic Finite Elements. Ph.D. thesis, FU Berlin (2015)

14. Hatcher, A.: Algebraic Topology. Cambridge University Press (2002)

15. Karcher, H.: Riemannian center of mass and mollifier smoothing. Commun. Pure Appl. Math. 30, 509-541 (1977)

16. Kobayashi, S., Nomizu, K.: Foundations of Differential Geometry, vol. II. Wiley (1969)

17. Sander, O.: Geodesic finite elements of higher order. IMA J. Numer. Anal. 36, 238-266 (2016)

18. Ur Rahman, I., Drori, I., Stodden, V.C., Donoho, D.L., Schröder, P.: Multiscale representations for manifold-valued data. Multiscale Modeling Simul. 4(4), 1201-1232 (2005)

19. Wallner, J.: Smoothness analysis of subdivision schemes by proximity. Constr. Approx. 24, 289-318 (2004)

20. Wallner, J.: On convergent interpolatory subdivision schemes in Riemannian geometry. Constr. Approx. 40, 473-486 (2014)

21. Wallner, J., Dyn, N.: Convergence and $C^{1}$ analysis of subdivision schemes on manifolds by proximity. Comput. Aided Geom. Des. 22, 593-622 (2005)

22. Wallner, J., Nava Yazdani, E., Grohs, P.: Smoothness properties of Lie group subdivision schemes. Multiscale Model. Simul. 6, 493-505 (2007)

23. Wallner, J., Nava Yazdani, E., Weinmann, A.: Convergence and smoothness analysis of subdivision rules in Riemannian and symmetric spaces. Adv. Comput. Math. 34, 201-218 (2011)

24. Xie, G., Yu, T.P.Y.: Smoothness equivalence properties of interpolatory Lie group subdivision schemes. IMA J. Numer. Anal. 30(3), 731-750 (2010)

Publisher's note Springer Nature remains neutral with regard to jurisdictional claims in published maps and institutional affiliations. 